\title{
VINCI-VLTI measurements of HR 4049: The physical size of the circumbinary envelope ${ }^{\star}$
}

\author{
S. Antoniucci ${ }^{1,2,3}$, F. Paresce ${ }^{3}$, and M. Wittkowski ${ }^{3}$ \\ 1 Università degli Studi di Roma "Tor Vergata”, via della Ricerca Scientifica 1, 00133 Roma, Italy \\ 2 INAF-Osservatorio Astronomico di Roma, via di Frascati 33, 00040 Monteporzio Catone, Italy \\ e-mail: antoniucci@mporzio.astro.it \\ 3 ESO-European Southern Observatory, Karl-Schwarzschild-Straße 2, 85748 Garching bei München, Germany
}

Received 22 June 2004 / Accepted 30 October 2004

\begin{abstract}
We present the first detection of the envelope which surrounds the post-AGB binary source HR 4049. VINCI-VLTI $K$-band interferometric observations of this source imply the existence of a large structure with a Gaussian angular FWHM $22.4 \pm 1.4$ mas or uniform disk diameter of $34.9 \pm 1.9$ mas. With the Hipparcos parallax of $1.50 \pm 0.64$ mas these values correspond to a physical size of $14.9_{-4.4}^{+11.1} \mathrm{AU}$ and $23.3_{-7.0}^{+17.3} \mathrm{AU}$, respectively. Our measurements, covering an azimuth range of $\sim 60^{\circ}$ for the sky-projected baseline, provide information on the geometry of the emitting region and show that there is only a slight variation of the measured angular values along the different directions sampled. Thus, our results are consistent with a spherical geometry of the envelope. However, we cannot completely rule out the existence of an asymmetric envelope (like the circumbinary disk envisaged by some recent models) because of the limited spatial frequency and azimuth range covered by the observations.
\end{abstract}

Key words. techniques: interferometric - circumstellar matter - stars: AGB and post-AGB - stars: evolution

\section{Introduction}

HR 4049 (HD 89353, AG Ant) is considered the prototype of a peculiar group of sources in the post-Asymptotic Giant Branch (AGB) stage of evolution that are believed to be close binary systems surrounded by a massive and stable circumbinary envelope as a consequence of mass loss processes during the AGB phase. These sources typically show evidence of extremely metal-depleted photospheres, possibly the result of dust formation in the circumstellar environment followed by re-accretion of the depleted gas onto the stars. In the case of HR 4049 the presence of large amounts of circumstellar dust is indicated both by the UV deficiency and strong IR excess (Lamers et al. 1986). At the moment, however, the size and spatial distribution of this material are not well known.

Several models of HR 4049 have been proposed, mainly based on photometric and polarimetric observations, with different predictions on the geometry and extent of the dust envelope, depending also on the physical properties of the dust grains. Waters et al. (1989) suggested, for instance, that the observed IR excess could be modelled by the emission from an optically thin cloud with a radius ranging from 5 to about $300 \mathrm{AU}$ or alternatively by a blackbody-emitting structure with a size of the order of 2 AU. More recent work on HR 4049

* Based on public shared risk science program data released from the ESO VLTI: http://www.eso.org/projects/ vlti/instru/vinci/vinci_data_sets.html tended to favour the flattened or asymmetric disk geometry for the distribution of the dust around the source. Photometric variations in phase with variations of radial velocity indeed suggest that the circumstellar matter is distributed in a disk surrounding the binary, which would be observed from Earth at a high inclination with respect to the plane of the sky (Waelkens et al. 1991). Analysis of the spectral energy distribution (SED) of HR 4049 has shown that the infrared excess between 1 and $850 \mu \mathrm{m}$ can be fitted almost perfectly with a single blackbody function at $T \sim 1150 \mathrm{~K}$. On this basis, it has been suggested that the radiation we observe is coming from the inner rim of a circumbinary disk heated by the central sources (Dominik et al. 2003). Moreover, polarization measurements indicate the presence of Rayleigh scattering by two different populations of grains, suggesting that the distribution of the circumstellar material might be asymmetric with the presence of a disk and a bipolar structure (Joshi et al. 1987; Johnson \& Jones 1991; Johnson et al. 1999).

In this paper, we present the analysis of VLTI-VINCI observations intended to shed light on this interesting problem by measuring the size and shape of the circumstellar envelope of HR 4049 directly by interferometric means. The observations clearly resolve for the first time a relatively large structure around the source with a dimension and shape that is quite plausibly attributable to the putative circumbinary envelope surrounding the binary. 
Table 1. Observational log with nights, baselines and sky-projected baseline azimuth for VINCI observations of HR 4049.

\begin{tabular}{lllll}
\hline \hline Night & \multicolumn{2}{c}{ VLTI baseline } & Proj. baseline (m) & Azimuth \\
\hline $2002-12-16$ & B3-C3 & $8 \mathrm{~m}$ & $7.4-7.5$ & $53^{\circ}-62^{\circ}$ \\
$2003-01-13$ & B3-C3 & $8 \mathrm{~m}$ & $7.8-7.9$ & $75^{\circ}-76^{\circ}$ \\
$2003-01-15$ & B3-C3 & $8 \mathrm{~m}$ & $7.8-8.0$ & $64^{\circ}-76^{\circ}$ \\
$2003-01-16$ & B3-C3 & $8 \mathrm{~m}$ & 7.9 & $74^{\circ}-75^{\circ}$ \\
$2003-01-20$ & B3-C3 & $8 \mathrm{~m}$ & $7.5-7.8$ & $77^{\circ}-80^{\circ}$ \\
$2003-02-25$ & B3-D1 & $24 \mathrm{~m}$ & $11.3-12.3$ & $105^{\circ}-108^{\circ}$ \\
\hline
\end{tabular}

\section{Observations and data reduction}

The observations (see Table 1 for a summary) have been carried out with the VLT Interferometer (e.g. Glindemann et al. 2003) between December 2002 and February 2003 using the near-infrared $K$-band VLTI commissioning instrument VINCI (e.g. Kervella et al. 2003), which recombines the light coming from two $0.35 \mathrm{~m}$ aperture siderostats (Derie et al. 2003). The data were taken in the framework of the VLTI shared risk science program and are publicly available from the ESO Science Archive. The two interferometric baselines B3-C3 and B3-D1 were used, which have a ground length of 8 and $24 \mathrm{~m}$, respectively, and the same ground position angle (East of North) of $71^{\circ}$.

With this configuration, several series of interferograms (consisting of 100, 200 and 500 scans) were obtained on HR 4049, with a fringe frequency of $60 \mathrm{~Hz}$. This rather slow frequency had to be chosen because of the relatively low $K$-magnitude of the object ( $K=3.21 \pm 0.29$, Cutri et al. 2003). The details about the calibrator stars used for the observations are shown in Table 2. The fringe frequency for several calibrators was $290 \mathrm{~Hz}$, different from the one used on the science target. This may introduce a calibration error which has been taken into account. By comparing the results obtained using only calibrators sampled with the same fringe frequency as the target (whenever possible) to those derived using all available calibrators, we have estimated the effect of this error on the calibrated visibilities to be less than $3 \%$.

Squared coherence factors were calculated processing the raw interferograms with the VINCI data reduction software (version 3.0) by Kervella et al. (2004). Subsequent calibration of the data and calculation of broad-band synthetic visibility values were performed as in Wittkowski et al. (2004).

A total of 17 values for the squared visibility were finally obtained from data reduction at an effective wavelength of $\lambda_{\text {eff }}=2.21 \mu \mathrm{m}$ and in an azimuth range for the sky-projected baseline between $53^{\circ}$ and $108^{\circ}$ (North through East).

We use two simple two-component models for the object intensity distribution, both composed of a single central star (the primary of the binary system) sourrounded by an envelope. For the central star, we use the radius $R_{*}=47 R_{\odot}$ derived by Bakker et al. (1998) and adopt an almost unresolved uniform disk (UD) brightness distribution with angular diameter 0.7 mas (on the basis of the Hipparcos-determined distance). Since the flux coming from the source is mainly due to the primary (e.g. Bakker et al. 1998) we do not take into account the much fainter secondary which has been detected by radial velocity measurements (Waelkens et al. 1991;
Table 2. Calibrator stars. The uniform disk diameter $\theta_{\mathrm{UD}}$ and the corrisponding error $\sigma_{\theta}$ are reported together with spectral type, $K$ magnitude and effective temperature.

\begin{tabular}{llrccl}
\hline \hline Star & Sp. type & $K$ mag & $\begin{array}{c}\theta_{\mathrm{UD}} \\
\text { mas }\end{array}$ & $\begin{array}{l}\sigma_{\theta} \\
\text { mas }\end{array}$ & $\begin{array}{l}T_{\text {eff }} \\
\text { K }\end{array}$ \\
\hline $31 \mathrm{Ori}^{a}$ & K5 III & 0.90 & 3.56 & 0.057 & 4046 \\
$51 \mathrm{Ori}^{a}$ & K1 III & 2.10 & 1.87 & 0.021 & 4508 \\
$\alpha \mathrm{Hya}^{b}$ & K3II-III & -1.21 & 9.11 & 0.911 & 4230 \\
$\delta \mathrm{Lep}^{a}$ & K0 III & 1.37 & 2.56 & 0.041 & 4656 \\
$\epsilon \mathrm{Lep}^{a}$ & K5 IIIv & -0.20 & 5.91 & 0.064 & 4046 \\
$\iota \mathrm{Hya}^{a}$ & K2.5 III & 0.91 & 3.41 & 0.048 & 4318 \\
$v_{2} \mathrm{CMa}^{a}$ & K1.5 III/IV & 1.62 & 2.38 & 0.026 & 4497 \\
$\mu \mathrm{Hya}^{b}$ & K4 III & 0.40 & 4.55 & 0.455 & 4090 \\
v337 Car $^{a}$ & K2.5 II & 0.03 & 5.09 & 0.058 & 4300 \\
\hline
\end{tabular}

${ }^{a}$ From Bordé et al. (2002), A catalogue of calibrator stars for LBSI.

${ }^{b}$ From calibration by Dyck et al. (1996) (Radii and Effective Temperatures for $\mathrm{K}$ and $\mathrm{M}$ Giants and Supergiants), using $K$ magnitudes from Gezari (1999) (Catalog of IR observations).

Bakker et al. 1998). As for the envelope, we describe it with an UD and a Gaussian distribution. We assume a ratio $F=$ $F_{*} / F_{\text {tot }} \sim 15 \%$ for the stellar flux with respect to the total observed flux in the $K$-band (Dominik et al. 2003). Keeping the adopted parameters of the (unresolved) star constant and treating the UD diameter or the Gaussian FWHM of the envelope as the only free parameter, we compare the observed visibility with our simple model.

Although these simple two-component models certainly represent a first approximation, this procedure can provide us basic information on the shape and dimensions of the emitting region in HR 4049 without having to resort to more complex and uncertain assumptions. We should bear in mind that interferometer observations carried out at different azimuth values for the projected baseline allow us, in principle, to detect asymmetries of the intensity distribution.

For this reason, we grouped the data points in four different $10^{\circ}$ wide azimuth bins $\left(53^{\circ}-62^{\circ}, 63^{\circ}-72^{\circ}, 73^{\circ}-82^{\circ}\right.$ and $103^{\circ}-112^{\circ}$ ). Then, we performed a best-fit of our models to the squared visibility values for each bin to obtain the corresponding values of the Gaussian FWHM and UD diameter of the envelope (see Fig. 1 and Table 3).

We also investigated how the obtained results vary depending on the adopted flux ratio $F$. We found that, when changing $F$ by $5 \%$, the related variation for the envelope angular size is small: about $5 \%$ and $4 \%$ for the Gaussian and UD distribution, respectively.

Finally, although the decrease of the observed visibility between spatial frequencies $\sim 16$ and $\sim 26$ cycles/arcsec clearly shows that we are resolving a compact envelope component, a contribution to the total visibility by an additional extended scattering component is quite possible. In this case, assuming that $10 \%$ of the total flux is due to this incoherent component, we found that the fitted sizes of the envelope (UD and Gaussian) decrease by roughly $10 \%$.

The measurements show that we have detected an emitting structure whose azimuthally averaged angular size is $22.4 \pm$ 1.4 mas (Gaussian $F W H M$ ) or $34.9 \pm 1.9$ mas (UD), with errors 
Table 3. VLTI-VINCI measurements of the size of the envelope surrounding HR 4049. Depending on the adopted source model (UD + Gauss or UD + UD, see the text), the FWHM of a Gaussian distribution or an equivalent uniform disk diameter $\theta_{\mathrm{UD}}^{\text {env }}$ are reported. Formal errors are shown; further error $(\sim 3 \%)$ is introduced by the calibration process (see the text). The measurements refer to the four azimuth bins mentioned in the paper and to a value $F=15 \%$ of stellar to total flux ratio. The last row shows the results when considering all data together.

\begin{tabular}{l|l|l}
\hline \hline Azimuth & UD + Gauss & UD+UD \\
\hline Bins & $F W H M \pm \sigma($ mas) & $\theta_{\text {UD }}^{\text {env }} \sigma($ mas $)$ \\
\hline $53^{\circ}-62^{\circ}$ & $22.7 \pm 0.1$ & $36.9 \pm 0.1$ \\
$63^{\circ}-72^{\circ}$ & $23.3 \pm 0.4$ & $37.7 \pm 0.6$ \\
$73^{\circ}-82^{\circ}$ & $23.8 \pm 0.6$ & $38.4 \pm 0.8$ \\
$103^{\circ}-112^{\circ}$ & $21.7 \pm 0.2$ & $33.4 \pm 0.4$ \\
\hline all azimuths & $22.4 \pm 0.5$ & $34.9 \pm 0.7$ \\
\hline
\end{tabular}

including also the previously mentioned uncertainties due to calibration and to the adopted flux ratio. There is only a slight variation of the size depending on baseline orientation, most of it when passing from the first three adjacent bins to the fourth one; the values range from 21.7 to 23.8 mas for the Gaussian and from 33.4 to 38.4 mas for the UD distribution. This indicates that our observations are consistent with a roughly spherical symmetric envelope; however, having measurements for only two different baselines, and therefore in a limited range of spatial frequencies and position angles, does not allow us to definitely rule out at this point the possibility that the envelope might be partly asymmetric and hence to unambiguously discriminate between a disk structure or a more spherical geometry. A more flattened envelope could explain the small variability of the object in the $K$-band (about $0.1 \mathrm{mag}$ in time scales of the order of 250 days, Waelkens et al. 1991); this would be due to different extiction along varying lines of sight to the primary as the components orbit each other (Waelkens et al. 1991). Because of our limited data sample we can only give here an estimate of the envelope size and we cannot exclude the existence of more complex structures consisting of several clouds or regions with different optical depth.

With the Hipparcos parallax of $1.50 \pm 0.64$ mas (Perryman et al. 1997) the azimuthally averaged angular values obtained for the Gaussian FWHM and the UD diameter of the envelope (Table 3) correspond to linear dimensions of $14.9_{-4.4}^{+11.1} \mathrm{AU}$ and $23.3_{-7.0}^{+17.3}$ AU, respectively.

\section{Discussion}

The geometry and extension shown by our results are in agreement with those proposed by Waters et al. (1989), who modelled the emission from HR 4049 as arising from a simple spherical optically thin envelope and found that such structure would have a radius ranging from $5 \mathrm{AU}$ up to about $300 \mathrm{AU}$.

Joshi et al. (1987) suggested that the observed intrinsic polarization of light from HR 4049 is introduced by scattering due to the circumstellar matter and that the low degree of polarization measured probably indicates that the envelope is almost spherically symmetric, which would be in agreement with our results. They modelled the strong dependence of the position angle with wavelength assuming the presence of two spheroidal dust shells surrounding the source, having different symmetry axis and composed by two different populations of large $(a \sim 1 \mu \mathrm{m})$ and smaller grains $(a \leq 0.05 \mu \mathrm{m})$.

Johnson \& Jones (1991) modelled the polarization as arising from scattering by dust in an envelope having the shape of a prolate ellipsoid; they found a position angle of about 8 degrees, remarking how this angle is approximately perpendicular to the direction of an extended (about $3 \mathrm{pc}$ ) emission at $100 \mu \mathrm{m}$ reported by Waters et al. (1989), presumably due to cold matter ejected from the source during the previous evolutionary stage.

It is also of interest to compare our interferometric results with the model proposed by Dominik et al. (2003) in which the emission we observe would actually come from the inner surface of a geometrically (Height/Radius $\sim 1 / 3$ ) and optically thick circumbinary disk surrounding the central sources and seen at an inclination of $\sim 60^{\circ}$ with respect to the plane of the sky. Using this model, the authors infer a radius of order $R \sim 10 \mathrm{AU}$ for the distance of the inner surface of the disk from the star, which is roughly consistent with the size of the structure detected by VINCI. Because of the high inclination, however, we would expect to observe a somewhat larger variation in the measured size of the structure for different baseline orientations. Assuming that we are observing in an azimuth range around the major axis of the projected disk (this would reproduce the trend we observe in our measurements, with the smallest values for the first and last bin), we should in fact see a diameter variation of at least 4 mas, which is around the $3 \sigma$ level in our measurements.

Finally, Johnson et al. (1999) proposed that the measured optical polarization indicates Rayleigh scattering arising in an optically and geometrically thin circumbinary disk. This, together with varying extinction with orbital phase, has led the authors to formulate the hypothesis that the orientation of the disk is tied to the orbit of the binary and so the projected face of the disc would change as the central sources orbit each other. Moreover, the UV polarization shows a typical $90^{\circ}$ change in position angle, usually related to the presence of a bipolar structure (i.e. two structures orientated perpendicularly to each other), which is often seen in planetary nebulae. In their measurements, the polarized light shows two preferential position angles (averaged over wavelength) of $\sim 17^{\circ}$ and $\sim 28^{\circ}$. It is interesting to notice that, since the observed PA of scattered light is supposed to be perpendicular to the plane of the circumstellar material in the disk, the orientation angle of the long axis of the disk, as viewed from Earth, would lie in the range $110^{\circ}-120^{\circ}$. Thus, the VINCI observations presented here (with $\alpha \sim 50^{\circ}-110^{\circ}$ ) would sample directions ranging from approximately that of the (projected) disk's long axis $\left(\alpha \sim 110^{\circ}\right)$ to one almost perpendicular. However, our results show that there is no clearly evident difference in the source dimensions when passing from one azimuth bin to the others, as one might expect assuming the disk configuration reported above.

Although HR 4049 is generally believed now to be surrounded by a circumbinary disk on the basis of the different observational evidences mentioned above, the existence of a more spherical envelope should not be excluded out of hand, 

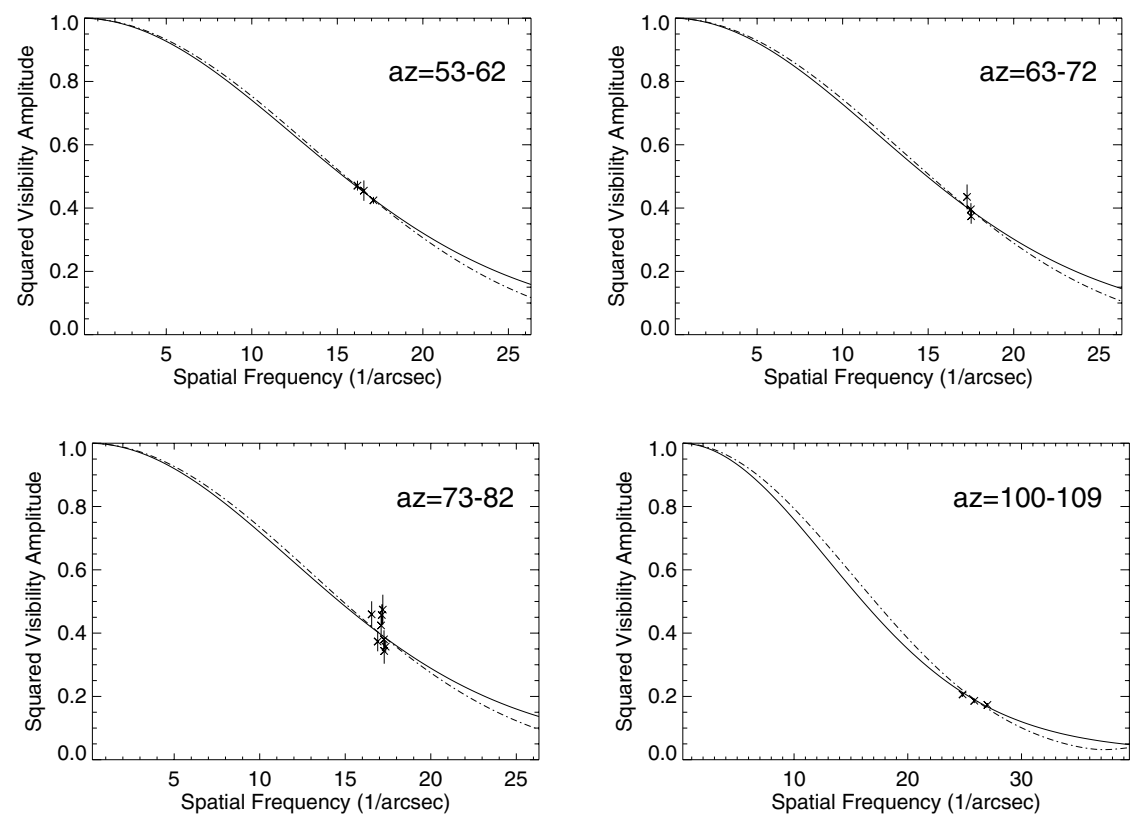

Fig. 1. Best-fit through the observed visibility points of the normalized squared visibility curve provided by the simple models of HR 4049 described in the paper. The solid curve corresponds to the UD + Gaussian (star + envelope) model, the dashed-dotted line to the UD + UD model, both for a stellar to total flux ratio $F=15 \%$. The four panels refer to the different bins of azimuth (see Table 3)

as the recent example of the Herbig AeBe stars clearly shows (e.g. Millan-Gabet et al. 2001).

Moreover, it is also likely that the envelope around this source might be multi-structured, as the polarization data suggest (bipolar structure, different populations of grains in separated shells).

\section{Conclusions}

The reduction and analysis of VINCI data on HR 4049 have led to the detection of a structure of angular dimensions 34.9 mas (uniform disk diameter) or 22.4 mas ( $F W H M$ of a Gaussian distribution), probably the circumstellar envelope or disk surrounding this binary system.

Assuming the Hipparcos-determined distance of $667 \mathrm{pc}$ for the source, this angular values corresponds to a size of about 23.3 AU and 14.9 AU respectively.

The measured size shows only a slight variation along different directions spanning an azimuth range of $\sim 60^{\circ}$, consistent with models of spherically symmetric or ellipsoidal envelopes (e.g. Waters et al. 1989; Joshi et al. 1987; Johnson \& Jones 1991).

We cannot, however, completely rule out the possibility of the presence of asymmetric structures, such as the circumbinary disk predicted by some recent models.

Our knowledge of the geometry of HR 4049 system can be refined with further interferometric observations. Sampling different azimuths and spatial frequencies and also carrying out measurements at different wavelengths would definitely help in constraining the models, thus providing new information on both the geometry and physics of the HR 4049 circumstellar envelope detected by VINCI. This will indeed be possible with the advent of the MIDI and AMBER instruments and the auxiliary telescopes to the VLTI facility in the next year or so.

Acknowledgements. We would like to thank I. Percheron, O. L. Lupie, V. Roccatagliata and D. Fedele for their helpful advice. For

this work we have made use of NASA's Astrophysics Data System Bibliographic Services and of the SIMBAD database, operated at CDS, Strasbourg, France.

\section{References}

Bakker, E., Lambert, D., van Winckel, H., et al. 1998, A\&A, 336, 263 Bordé, P., Coude du Foresto, V., Chagnon, G., \& Perrin, G. 2002, A\&A, 393, 183

Cutri et al., 2MASS All-Sky Catalog of Point Sources, University of Massachusetts and IPAC/California Institute of Technology

Derie, F., Brunetto, E., Duchateau, M., et al. 2000, Proc. SPIE, 4006, 99

Dominik, C., Dullemond, C. P., Cami, J., \& van Winckel, H. 2003, A\&A, 397, 595

Dyck, H. M., Benson, J. A., van Belle, G. T., \& Ridgway, S. T. 1996, AJ, 111, 1705

Gezari 1999, Catalog of IR observations http://cdsweb.u-strasbg.fr/viz-bin/ VizieR?-source=II $/ 225$

Glindemann, A., Algomedo, J., Amestica, R., et al. 2003, Proc. SPIE, 4838,89

Johnson, J. J., \& Jones, T. J. 1991, AJ, 101, 1735

Johnson, J. J., Anderson, C. M., Bjorkman, K. S., et al. 1999, MNRAS, 306, 531

Joshi, U. C., Deshpande, M. R., Sen, A. K., \& Kulshrestha, A. 1987, A\&A, 181, 131

Kervella, P., Gitton, P. B., Segransan, D., et al. 2003, Proc. SPIE, 4838, 858

Kervella, P., Ségransan, D., \& Coudé Du Foresto, V. 2004, A\&A, 425, 1161

Lamers, H., Waters, L., Garmany, C., Perez, M., \& Waelkens, C. 1986, A\&A, 154, L20

Millan-Gabet, R., Schloerb, F. P., \& Traub, W. A. 2001, ApJ, 546, 358

Perryman, M. A. C., Lindegren, L., Kovalevsky, J., et al. 1997, A\&A, 323,49

Waelkens, C., Lamers, H., Waters, L., et al. 1991, A\&A, 242, 433

Waters, L., Lamers, H., Snow, T. P., et al. 1989, A\&A, 211, 208

Wittkowski, M., Aufdenberg, J. P., \& Kervella, P. 2004, A\&A, 413, 711 